\title{
System Simulation for EMC Network Analysis
}

\author{
M.Foissac, JL.Schanen, G.Frantz, D.Frey \\ G2ELab (UMR 5269 CNRS-INP-UJF) \\ Bat Ense3 BP 46 \\ F-38330 SMH Cedex Grenoble France \\ jean-luc.schanen@g2elab.grenoble-inp.fr
}

\author{
C.Vollaire \\ Ampere Lab - Ecole Centrale de Lyon \\ 36, avenue Guy de Collongue \\ F 69134 Ecully cedex \\ Christian.Vollaire@ec-lyon.fr
}

\begin{abstract}
This paper uses compact models of power electronics converters to represent their EMC behavior. The model does not need any knowledge of the converter design since it is identified from external measurements. Three different DC-DC converters will be identified and the model validated in various network environment. Then, the models association in a DC network will be studied.
\end{abstract}

\section{INTRODUCTION}

Determining the ElectroMagnetic Compatibility (EMC) behavior of any electrical system is always a challenging task for the designers, since it covers a wide frequency range, from some 10th of hertz to several 10 th of $\mathrm{kHz}$ for conducted emissions. Up to now, to simplify the task of the system engineers, this problem is addressed using emission and susceptibility standards [1]. However, meeting these standards requires the development of EMC filters, often expensive, heavy and bulky (up to $30 \%$ of the converter volume and cost). Since system optimization is more and more required, especially in embedded network as aircraft applications [2], it becomes necessary to change the approach, and not to design individual filters based on standards, but to achieve a real system analysis and to develop optimized filters adapted to the network requirements.

To reach this goal, one preliminary step is to be able to simulate the behavior a complete electrical network, including several power converters. It is not feasible to use the conventional power electronics simulators in the time domain: even if some of them may be able to provide interesting results. Indeed, simulation time and memory consumption are excessive. Therefore, a parametric study is not possible, and after simulation, the interpretation of the results is too complex. This temporal approach must be kept for validation purpose only.

For this reasons, other modeling methods have been proposed in the past years, working directly in the frequency domain and replacing the semiconductors by equivalent sources [3-4]. However these kinds of methods still necessitate the knowledge of the converter content, especially in term of stray elements. First, manufacturer not always agree to provide such data (internal layout, component references), what leads to difficulties in gathering all models. Second, the complete model of the network, which may include hundreds of converters, would be still huge, since all parasitic at converter level should be considered.

Therefore, there has been recently a new effort in proposing alternative modeling methods, leading to more compact EMC models [5-7]. The principle of one of these models will be reminded in section II, and applied to three DC-DC boost converters (14-42V), with different layouts in section III. Model validation will also be proposed. Finally, section IV will associate the converters models in a network simulation.

\section{II. "BLACK BOX" EMC MODEL}

As proposed in [5-7], the model uses a quadrupole representation, involving two current sources and three impedances (Fig.1). It accounts for the ground potential, what is mandatory for EMC study.

The model identification is based on several operating conditions, obtained by varying the line impedance, as illustrated in Fig.2. Using voltage and currents V1, V2, I1, I2 measured for each operating point, the model parameters can be deduced. Every operating point leads to two equations linking V1, V2 to I1, I2. Since the number of parameters in the model is 5, three different measurements must be achieved. Using five of the six equations leads to the model identification [5].

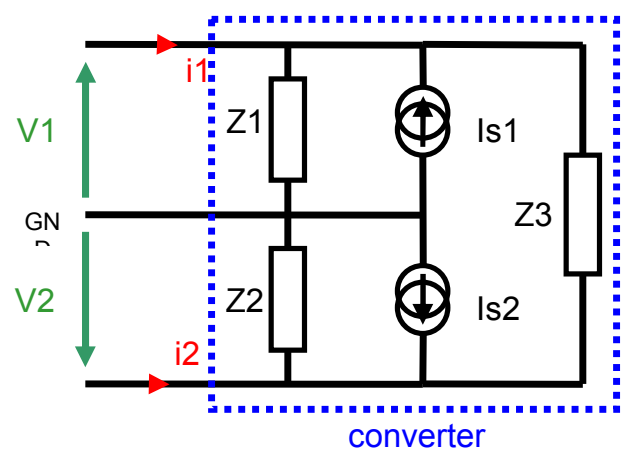

Fig. 1 : Model of a converter 


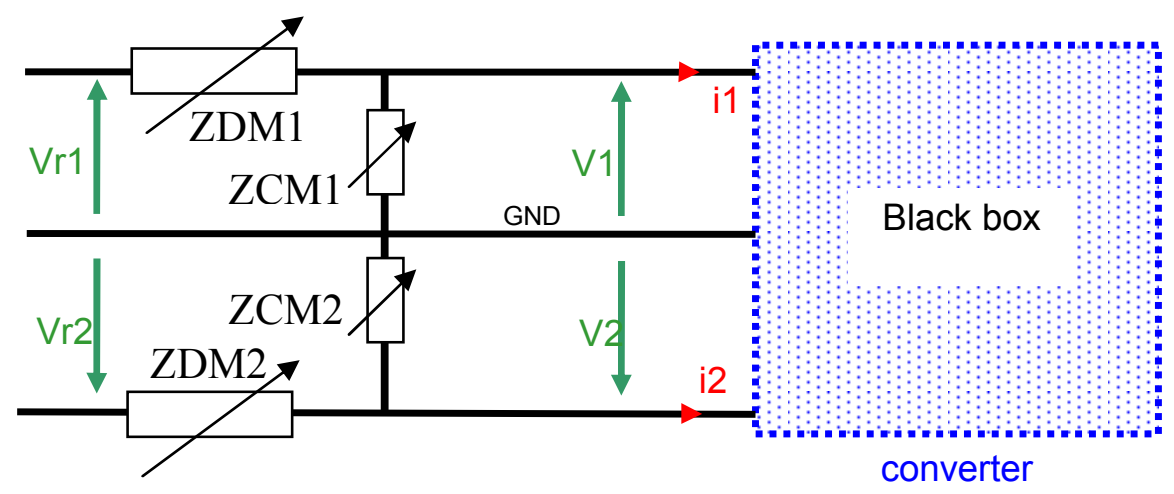

Fig. 2 : Line impedance variation for parameters identification

Even if it does not provide the best accuracy in the determination, the simplest way to identify the model has been chosen as follows:

- one operating condition with a direct connection between the DC source and the converter (undefined ZDM1, ZCM1, ZDM2, ZCM2)

- one operating condition with the "plus" potential connected to the ground $(\mathrm{ZMC} 1=0=>\mathrm{V} 1=0)$

- one operating condition with the "minus" potential connected to the ground $(\mathrm{ZMC} 2=0=>\mathrm{V} 2=0)$

The following equations describe briefly how the model parameters are derived from the three measurements (the subscript $\mathrm{i}$ for measured values $\mathrm{V} 1_{\mathrm{i}}, \mathrm{V} 2_{\mathrm{i}}, \mathrm{I} 1_{\mathrm{i}}, \mathrm{I} 2_{\mathrm{i}}$ indicates the Operating Condition)

Operating Condition 1

$\rightarrow I 1_{1}=\frac{V 1_{1}}{Z_{1}}-I_{s 1}+\frac{V 1_{1}-V 2_{1}}{Z_{3}}$
$\rightarrow I 2_{1}=\frac{V 2_{1}}{Z_{2}}-I_{s 2}-\frac{V 1_{1}-V 2_{1}}{Z_{3}}$

Operating Condition 2: $\mathrm{V} 1=0$

$\rightarrow I 1_{2}=-I_{s 1}+\frac{-V 2_{2}}{Z_{3}}$

$\rightarrow I 2_{2}=\frac{V 2_{2}}{Z_{2}}-I_{s 2}+\frac{V 2_{2}}{Z_{3}}$

Operating Condition 3: V2 $=0$

$\rightarrow I 1_{3}=\frac{V 1_{3}}{Z_{1}}-I_{s 1}+\frac{V 1_{3}}{Z_{3}}$

$\rightarrow I 2_{3}=-I_{s 2}-\frac{V 1_{3}}{Z_{3}}$

With 6 equations for 5 parameters (Z1, Z2, Z3, Is1, Is2), one of them is useless. It has been shown in [5] that it is better to keep the two equations of the operating condition 1, since it corresponds to a normal working of the converter. Therefore, equation (5) has been arbitrary removed, and the non linear system can thus be solved. Obviously, the quality of the signal acquisition is of great importance, since all the accuracy of the identification depends on it. The signals have been recorded in the time domain, with a large memory oscilloscope to keep a sufficient number of data points in the period. Indeed, a Fourier Transform is needed after this acquisition in order to extract the model parameters in the frequency domain. To be noted that the direct signal measurement in the frequency domain using a spectrum analyzer is not possible, since the signals composition in the equations necessitates the phase shift, which is not available with such device.

\section{IDENTIFICATION OF SEVERAL DC-DC CONVERTERS}

\section{A. Converters Description}

Three 100W DC-DC 14-42V boost converters have been built, using various layout and technologies. Two different layouts have been chosen, one with low common mode current emission capability, and one with a large floating point track, originating a high common mode current level. In the same idea, Printed Circuit Board (PCB) and Insulated Metal Substrate (IMS) have been used: IMS is highly capacitive and thus generates more common mode current. Fig. 3 shows the various converters used. They have their own control circuit, with a switching frequency roughly equal to $100 \mathrm{kHz}$ (between $102 \mathrm{kHz}$ and $116 \mathrm{kHz}$ depending on the converter).

\section{B. Converter Model Identification and Validation}

The identification of each converter has been achieved in a standard environment, using a Line Impedance Stabilisation Network (LISN) between the DC source and the converter. Fig. 4 shows an example of the experimental results for voltage and current during the identification (temporal acquisition and illustration in the frequency domain, using a FFT - modulus only).

After identification, it is possible to compute the voltage across the LISN using the "Black Box" model, and a model of the cable used between the LISN and the converter in Operating Condition 1 (the cable model has been obtained experimentally using impedance measurement). Fig.5. gives the comparison between this reconstructed voltage and the direct measurement on the LISN, which has been recorded in Operating Condition 1. This has been achieved for the three converters. 


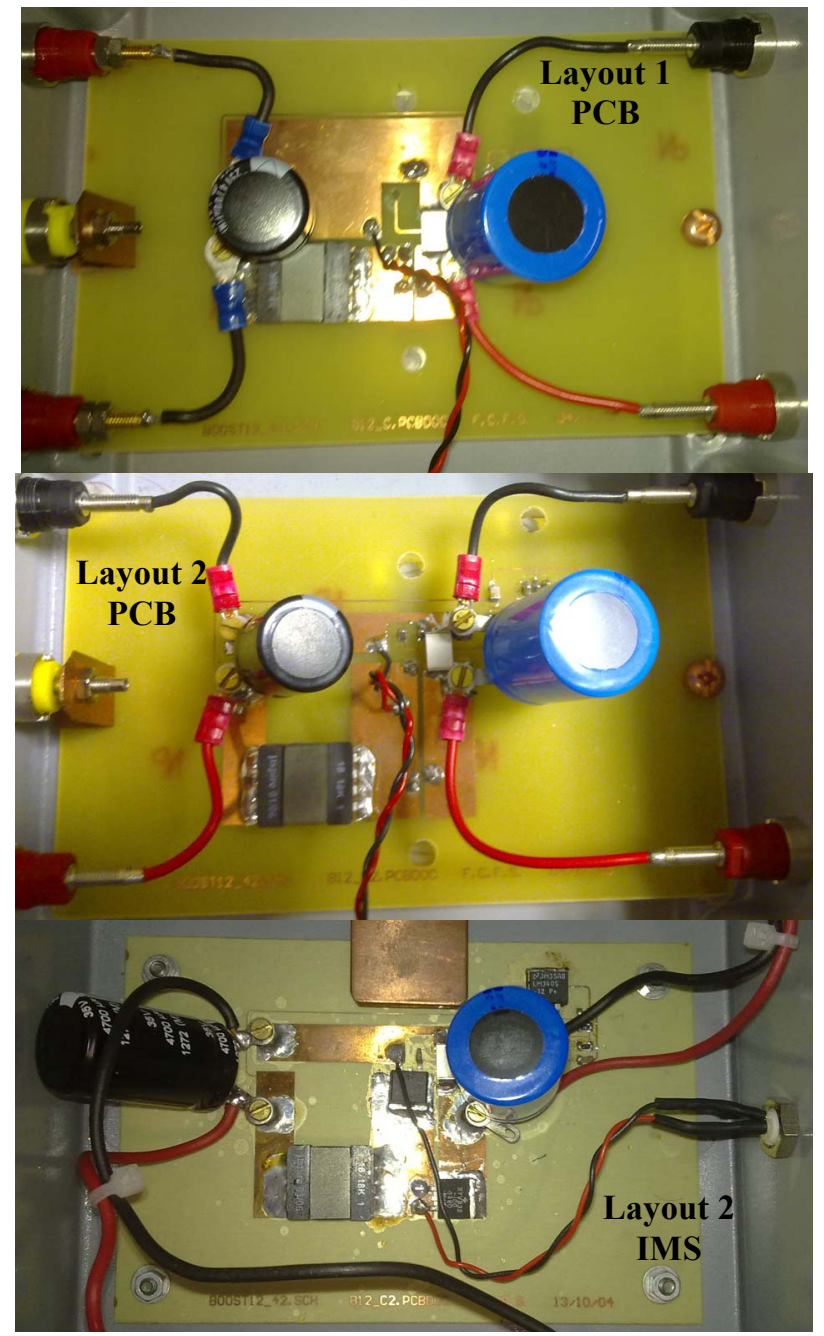

Fig. 3 : Three DC-DC converters with various layouts and technologies, and consequently different EMC behaviour.

This is not a real validation of the model since the LISN voltage has been measured in the same operating condition that the identification (i.e. for the same line impedance). However, it confirms that the identification has been correctly achieved, even if some imperfections can be noticed around $1 \mathrm{MHz}$ and in the high frequency range.

The errors around $1 \mathrm{MHz}$ have been attributed to external noise in the lab, which have been really hard to remove. A new measurement setup will be soon installed in an EMC chamber of the lab to avoid this phenomenon.

The high frequency inaccuracy can be mainly explained since the frequency of the boost oscillator was not stable during the different operating conditions. For some reasons, during the plus-ground and minus-ground short circuits, the switching frequency changed of about some percent. Therefore, the model identification was disturbed, since the harmonics of the three operating conditions were not at the same frequency. Additional errors may come from oscilloscope sensitivity which is perhaps not sufficient to reach small amplitude with a sufficient accuracy.
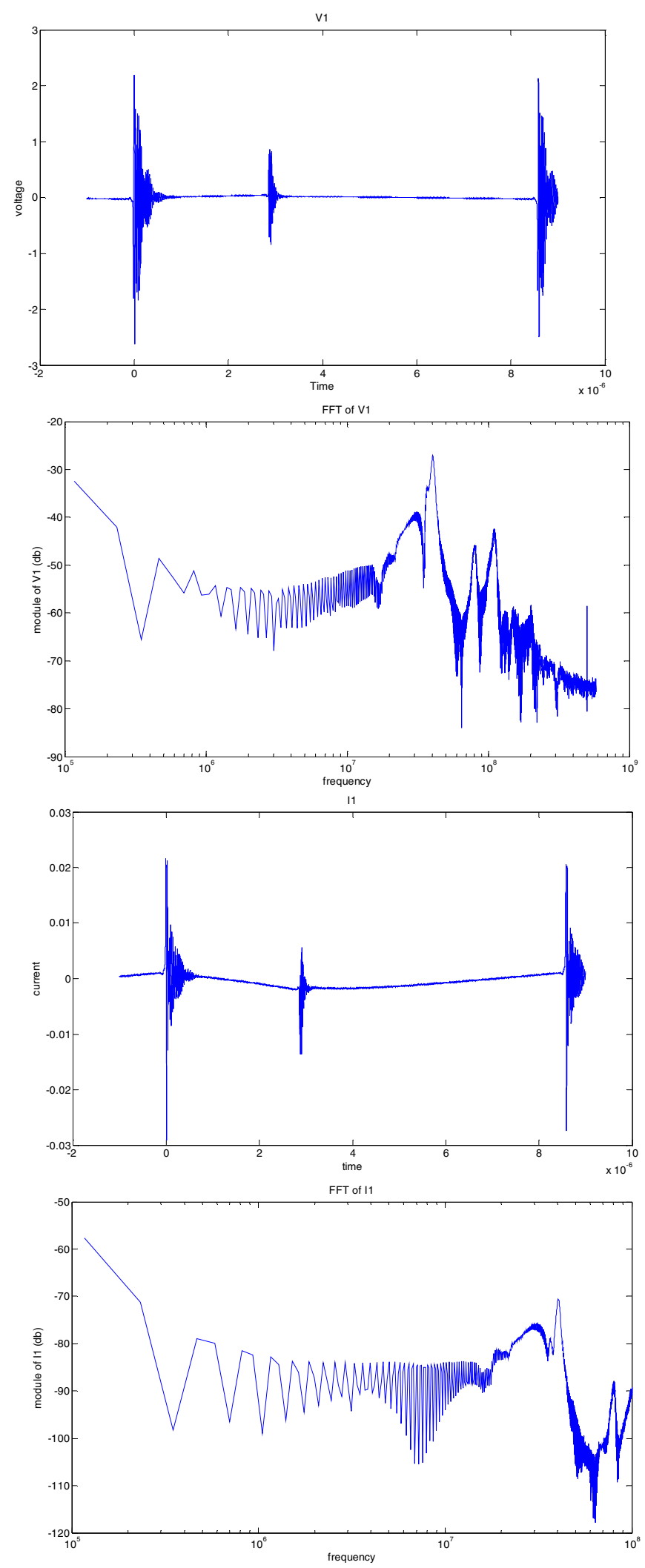

Fig. 4 : Example of voltage V1 (top) and current I1 (bottom) measurement used for identification. Time and Frequency domain (modulus only). 

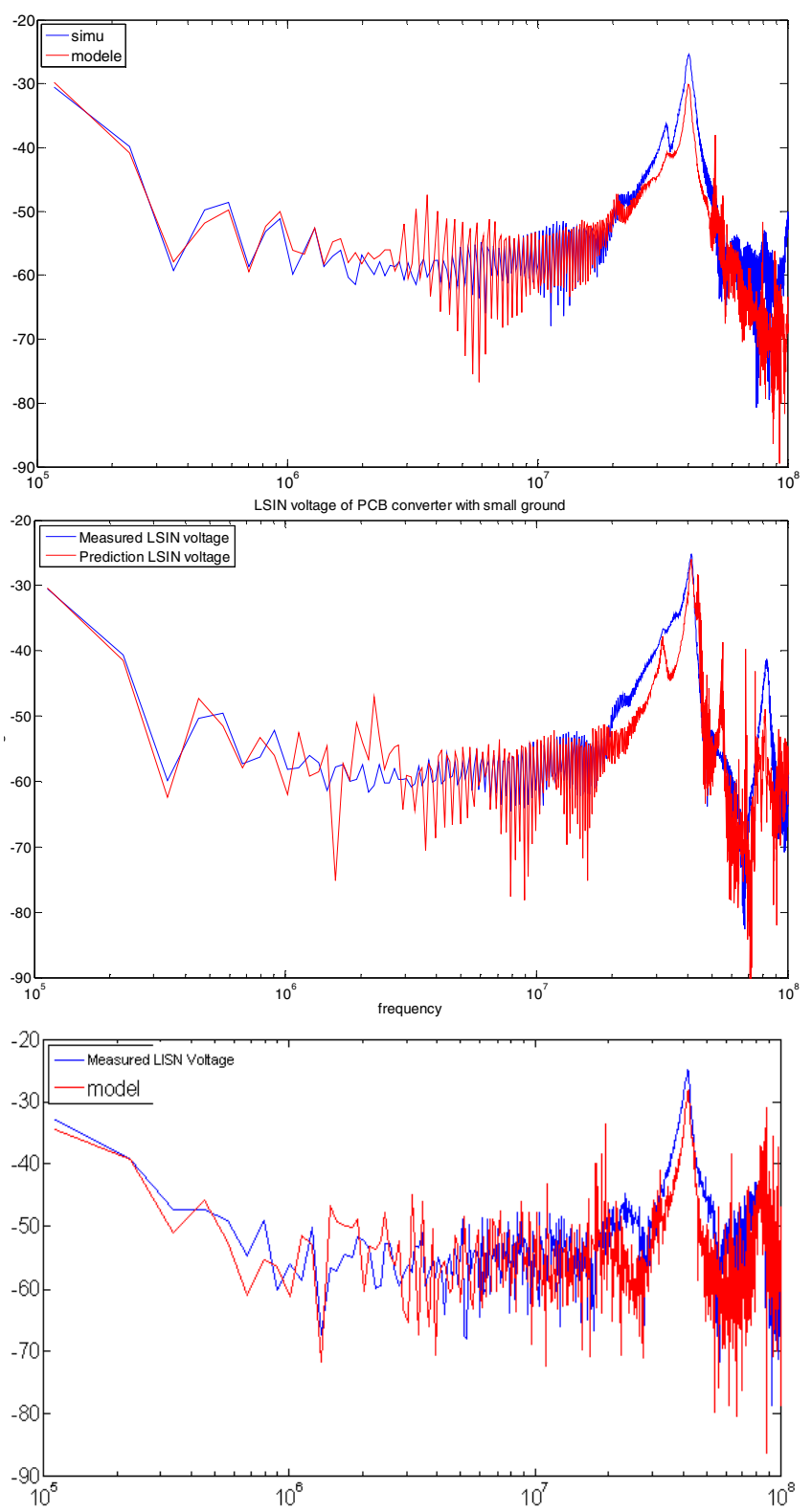

Fig. 5 : Comparison of the measured LISN voltage $[\mathrm{dB}]$ in Operating Condition 1 and the estimation based on the identified model for PCB layout 1 (top), PCB Layout 2 (Middle) and IMS Layout 2 (Bottom).

Finally, it can be noticed that the layout impacts on the LISN voltage in the high frequency range (over $50 \mathrm{MHz}$ ), since the spectrum of layout 2 increases, what is not the case for layout 1. The IMS technology results in a sharper resonance near $40 \mathrm{MHz}$.

The model has then been tested on a different network: the cable between the LISN and the converter has been changed. Conventional wires have been replaced by a shielded twisted pair. The LISN voltage has been recorded, and the model, used in combination with the model of the new cable, has been compared with the measurement. The result of this validation is given Fig. 6 for Layout 2 PCB.

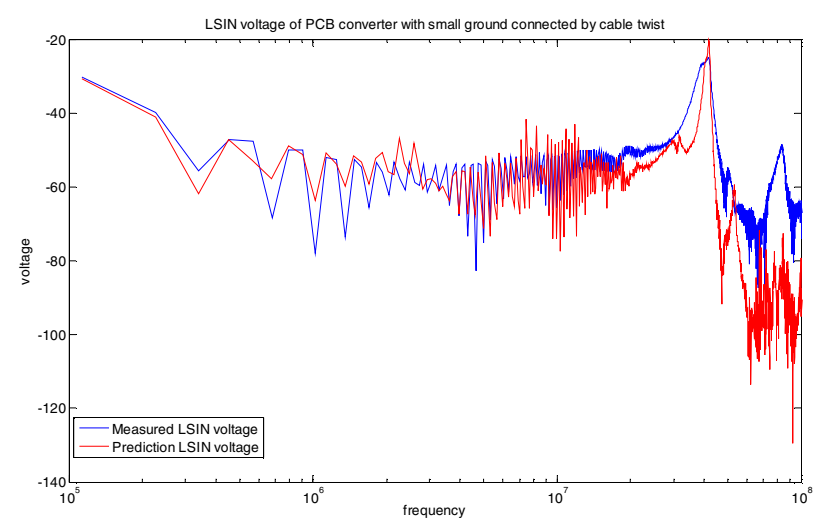

Fig. 6 : Comparison of the measured LISN voltage and the estimation based on the identified model with a different cable between the LISN and the converter (Layout $2 \mathrm{PCB}$ ).

The result is still fair, even if the high frequency behavior (over $50 \mathrm{MHz}$ ) could be improved. This may also be attributed to a bad estimate of the shielded twisted pair model.

Nevertheless, it appears that the modeling method can be used to reconstruct the EMI generated by a converter, even for different operating conditions than the one used for identification. Therefore, a combination of several "Black Box" models will be used to check the ability of this representation for EMC study in a DC network.

\section{APPLiCATION TO DC NETWORK}

A DC network has been built, using the two PCB converters and different cables. Using the models identified in the previous section, and cable models, it is possible to predict the LISN voltage at the DC network input. This approach is especially interesting since the two converters do not have strictly the same characteristics, and especially not the same switching frequencies. Therefore, the LISN signals are complex.

The first approach has been carried out using simulation. A Buck converter has been described using a quite accurate representation, with interconnects and components parasitics, and Pspice models of silicon devices (the diode has been built with a macro-block and a behavioural model to account for the recovery phenomenon).

The identification of the Buck converter has been achieved in simulation, using the same method presented in section II (short circuits). With the idealised "accuracy" of the simulation (contrary to oscilloscope measurement), no frequency variation between the different operating conditions, and no external disturbance, the simulated and reconstructed LISN voltage are perfect, as shown in Fig.7. When making a small change in the frequency between the different operating conditions (5\% in Op.Cond 2 and $10 \%$ in Op.Cond 3), the same kind of high frequency difference as encountered in measurement occurs (Fig. 8). This confirms the previous explanations for measurement inaccuracies. 


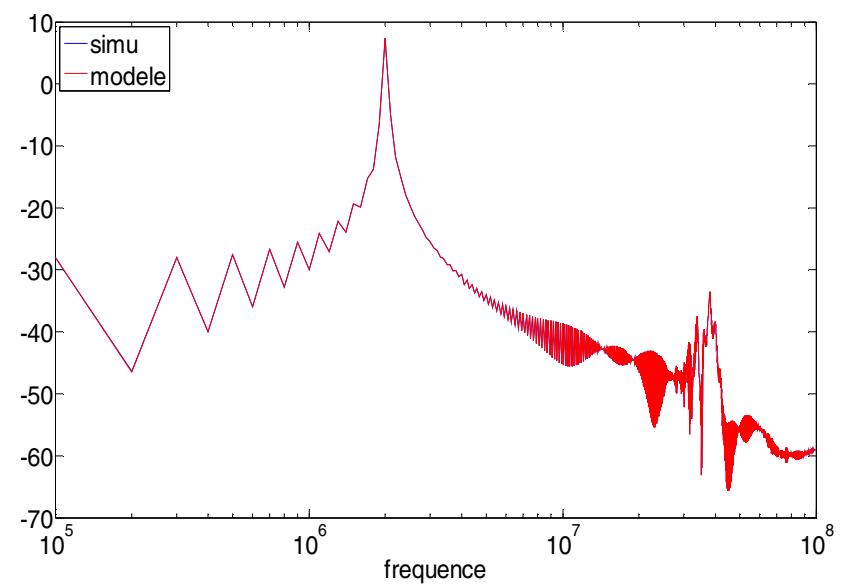

Fig. 7 : Comparison of the simulated LISN and the reconstructed result using the identified model. Simulation. Note the perfect correspondence since no external errors are present, contrary to measurement.

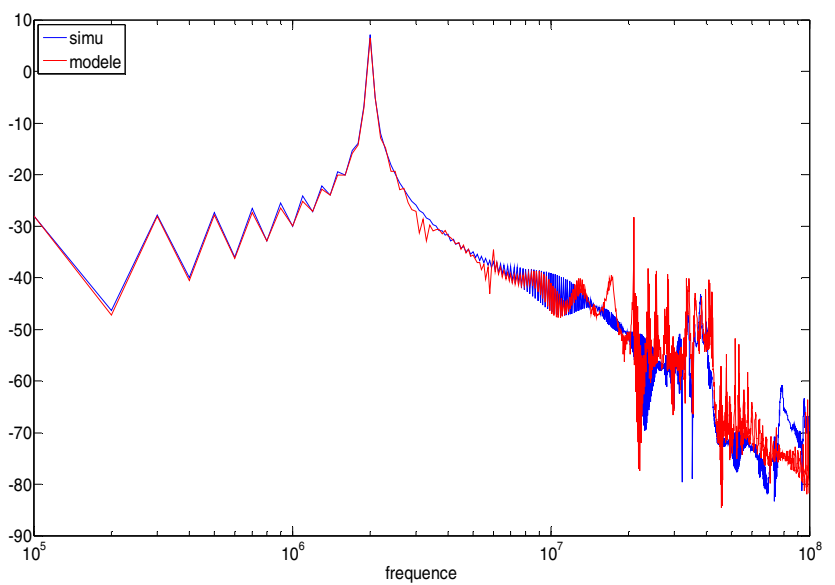

Fig. 8 : Comparison of the simulated LISN and the reconstructed result using the identified model. Simulation with 5\% variation for Op.Cond 2 and $10 \%$ for Op.Cond 3 .

After this preliminary study, two identical Buck converters have been joined in a simple network: one converter is linked to the LISN trough $500 \mathrm{nH}$ wire inductance, the other one connected in parallel with the first through another 500nH wire - Fig.9 Top.

However, as mentioned before, in the actual case, the converters switching frequencies are different. The effect of this difference has been thus checked in the simulation, to investigate its impact. Fig.10. shows the results for $100 \mathrm{kHz}$ and $110 \mathrm{kHz}$. The global shape is good, but some discrepancies appear for both low and high frequency range.

Some errors may be attributed to the bad estimate of the steady state (low frequency). Indeed, this case for time simulators is often tricky, with the low frequency component. Furthermore, it has not been possible to achieve the simulation and the FFT on a complete low frequency period $(10 \mathrm{kHz})$, due to the file size.

However, the global result is fair, and validates the proposed approach.

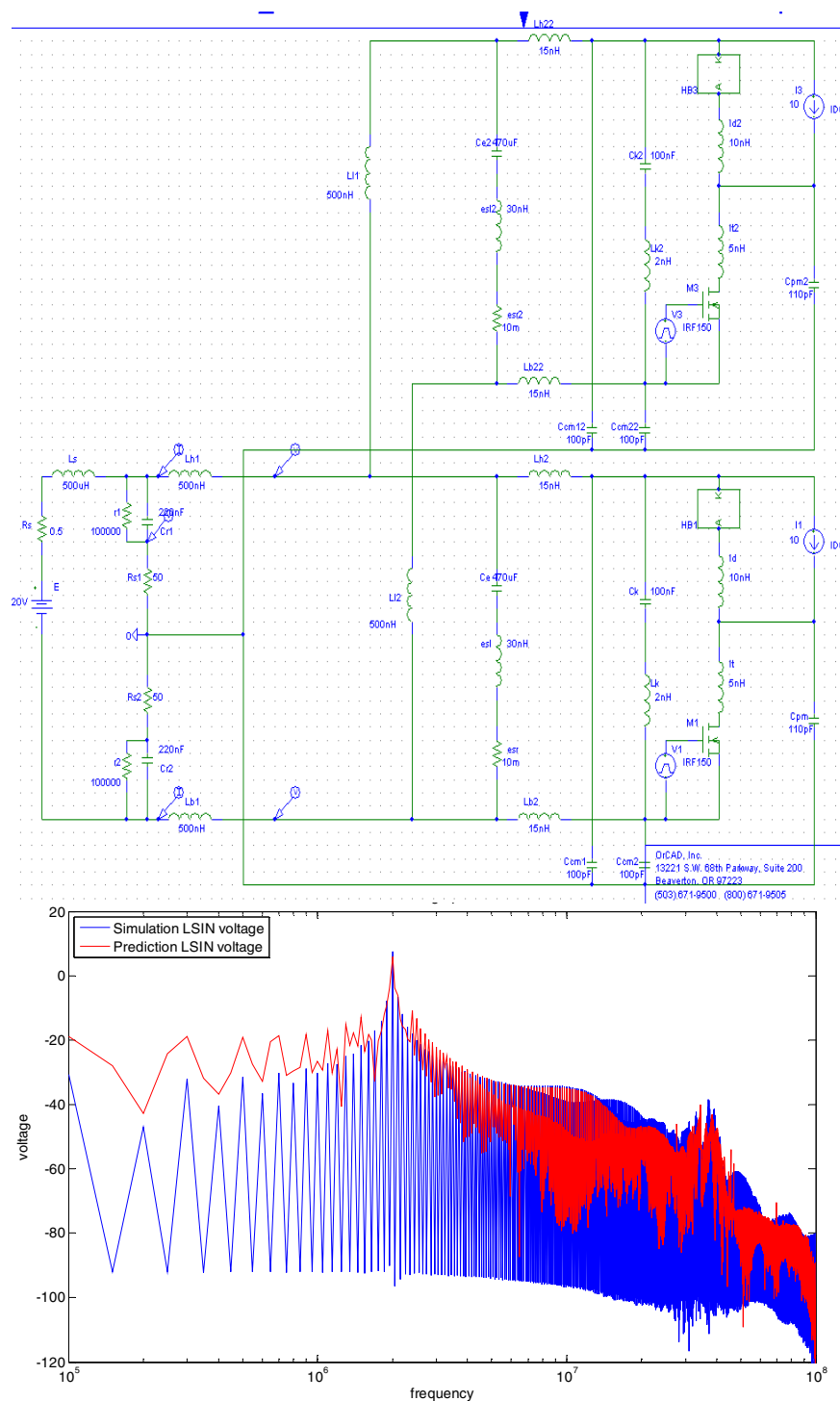

Fig. 9 : Comparison of the simulated and reconstructed LISN voltage for a two converter network. Converters are identical and switch at the same frequency.

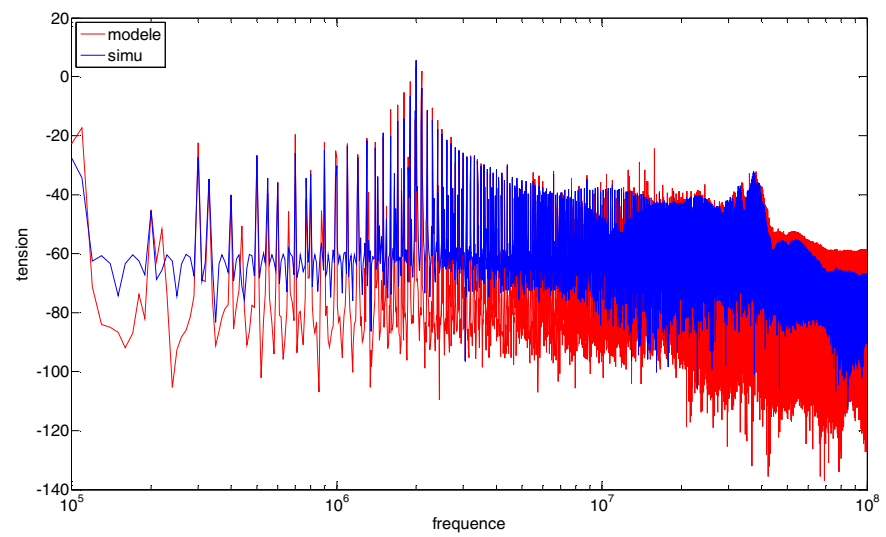

Fig. 10 : Comparison of the simulated and reconstructed LISN voltage for a two converter network. Converters are identical but switch at $100 \mathrm{kHz}$ and $110 \mathrm{kHz}$. 
An experimental approach has been then proposed, using the two PCB boost converters. They obviously switch at different frequencies. One converter has been connected to the LISN using known short wires, the second one through a shielded twisted cable (identified in section III). The LISN measurement has then been compared with the reconstruction using the boost models and the cable models. Fig. 11 shows the results.

Once again, the measurement is disturbed around $1 \mathrm{MHz}$ (external nose). A bad estimate of the $400 \mathrm{kHz}$ peak can be attributed to signal processing error during the signal acquisition. The rest of the curve is quite good.

Even if the measurement process used for identification and validation should be improved further, it seems that the proposed models can be used in a EMC network simulation. Obviously further validations with more complex cables must be undertaken, and the limits of the model checked: what are the extreme cable impedance values, for which the "Black Box" identification will become false, since the hypothesis of an equivalent source, not dependent from network impedance will no more be fulfilled...

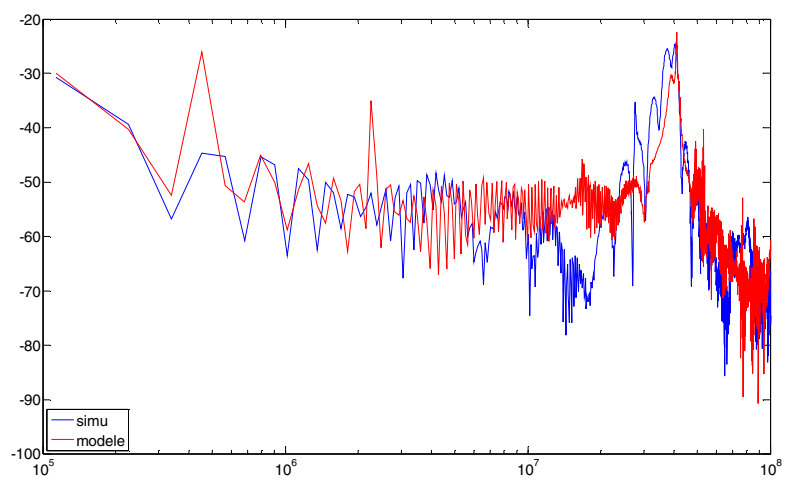

Fig. 11 : Comparison of the measured and reconstructed LISN voltage for a two converter network using two PCB converters.

\section{CONCLUSION}

The new paradigm in EMC is no more to design filters in order to meet standards, but to achieve a real estimate of the EMC behaviour of an electrical network. This is a mandatory step for filter and system optimization. The proposed "Black box" model and the associated identification method is an answer to this challenge. It has been validated experimentally on several DC-DC converters, realized with different technologies. Then, the association of all models in a small network has been investigated both in simulation and measurement. Even if the results should be improved, they are promising.

\section{REFERENCES}

[1] Yazar, M.N. Civilian EMC Standards and Regulations, Electromagnetic Compatibility, IEEE Transactions on, Volume EMC21, Issue 1, Feb. 1979

[2] More Open Electrical Technologies project (MOET): www.eurtd.com/moet/PDF/MOET\%20Publishable\%20Executive\%20 Summary.pdf

[3] B. Revol, J. Roudet, J.L. Schanen, P.Loizelet, "EMI study of a three phase inverter-Fed Motor Drives", IEEE IAS 04, 2-7 octobre 2004, seattle

[4] Jettanasen, C.; Costa, F.; Vollaire, C.; "Common-Mode Emissions Measurements and Simulation in Variable-Speed Drive Systems" Power Electronics, IEEE Transactions on, Volume 24, Issue 11, Nov. 2009

[5] Foissac, M.; Schanen, J.-L.; Vollaire, C.; "Black box" EMC model for power electronics converter, Energy Conversion Congress and Exposition, 2009. ECCE. IEEE, 20-24 Sept. 2009

[6] Baisden, A.C.; Boroyevich, D.; Wang, F.; "EMI Terminal Modeling"IAS'08, Edmonton, Canada.

[7] Jin Meng, Weiming Ma, "Noise Source Lumped Circuit Modeling and Identification for Power Converters", IEEE Transactions on industrials electronics, vol. 53, no. 6, decenbre 2006 